\title{
Gefahren unter der Sonne. Zum Verhältnis von Differenz und (Un-)Sicherheit am Beispiel europäischer Seeleute im kolonialen Kalkutta
}

\author{
Florian Neiske
}

\begin{abstract}
In October 1864, the Indian seaport Calcutta was hit by a heavy cyclone. The devastation that it caused to shipping left a large number of European seamen stranded in the city, without work and in some cases homeless. This triggered an intensifying debate among the colonial elites about the situation of these seamen that revolved around various security issues associated with this group. On grounds of the characteristics and behaviours attributed to them, they were perceived as a potential threat to, for instance, public order, but also to the ruling ideologies that were based on notions of Europe's putative civilisational superiority. At the same time, seamen were deemed to be a particularly vulnerable group. The Indian climate, various diseases and allegedly predatory groups were believed to be responsible for their high mortality rate as well as for their supposed moral decay. This article argues that this interaction between different conceptions of security and categorisations of difference revealed contradictions within the ruling ideologies. It was these contradictions that the colonial elites considered problematic and implicitly moved to the centre of the securitisation debate. As focussing on the debate alone would be insufficient to understand the colonial government's response in the aftermath of the cyclone, this article considers the position of the seaman within the economic system as well. Their position was marked by substantial insecurity, which the colonial administration was willing to tolerate as this served economic interests. The aforementioned contradictions thus resulted from the material conditions and ideological conceptions that were inscribed into the system of rule itself. To properly grasp processes of this kind, this article proposes a deeper integration of structural factors and societal analyses in the consideration of securitisation.
\end{abstract}




\section{Einleitung}

In den Morgenstunden des 5. Oktober 1864 wurden die Hafenstadt Kalkutta $^{1}$, Hauptstadt Britisch-Indiens, und weite Teile des bengalischen Umlandes von einem schweren Zyklon getroffen. Begleitet wurde der Sturm von einer Flutwelle, welche nicht nur unmittelbar an der Küste für Überschwemmungen sorgte, sondern sich bis in die Stadt selbst ausbreitete. Gemeinsam verursachten Sturm und Welle enorme Schäden und Verwüstungen. Wenngleich genaue Angaben in den ländlichen Gebieten Bengalens nur schwer zu ermitteln waren, kam ein späterer Bericht auf eine Anzahl von nahezu 50.000 Todesopfern durch die unmittelbaren Folgen des $\mathrm{Zy}$ klons (vgl. Gastrell/Blanford 1866). Hinzu traten Krankheiten, die sich noch Wochen später in den betroffenen Gebieten ausbreiteten. Schätzungen zufolge forderten diese weitere 25.000 bis 30.000 Menschenleben. Für Kalkutta und seine Vororte selbst sprechen die Berichte von insgesamt 49 Todesopfern durch den Sturm. Schwere Schäden wurden zudem an Infrastruktur und Gebäuden verzeichnet, vor allem in den ärmeren Vierteln der Stadt. Besonders betroffen waren darüber hinaus die Schifffahrt, Werften und Hafenanlagen. Aufgrund der Höhe und Stärke der Flutwelle riss diese zahlreiche Schiffe von ihren Vertäuungen und ließen diese kentern, ineinander treiben oder ans Ufer laufen. Von den 195 Schiffen, die sich am 5. Oktober im Hafen befunden hatten, wurden am folgenden Tag nur 23 ohne Schäden vorgefunden. 36 Schiffe galten gar als Totalverluste.

Infolge dieser enormen Schäden waren europäische ${ }^{2}$ Seeleute in groBer Zahl arbeits- und teilweise auch obdachlos in der Stadt gestrandet. Während man von offizieller Seite zunächst optimistisch gewesen war, mögliche Problematiken aufzufangen, indem Seeleute beispielsweise als zusätzliche Polizeikräfte eingesetzt wurden oder die Aufräum- und Reparaturarbeiten genug Arbeitsmöglichkeiten boten ${ }^{3}$, änderte sich dies mit dem Andauern der Situation. Durch ihre fortgesetzte Präsenz intensivierte sich eine Diskussion über die Lage und das Auftreten der Seeleute, welche sowohl innerhalb der Regierungsbehörden, aber auch in der Öffentlichkeit

1 Heutiger Name der Stadt ist Kolkata. Der Aufsatz folgt dem aktuellen wissenschaftlichen Standard, den Namen „Kalkutta“ (engl. 'Calcutta') zu benutzen, um bewusst zu betonen, dass die britische Kolonialstadt betrachtet wird.

2 In den Quellen bezieht sich der Begriff zum größten Teil auf britische Seeleute, schließt aber Seeleute anderer europäischer Nationen oder auch der USA ein. In diesem Sinne fungiert der Begriff nahezu als Synonym für „weiß“ und „christlich“.

3 Siehe V.H. Schalch an Secretary to the GovBengal, 17. Oktober 1864, No 1287, British Library (BL), India Office Records (IOR) IOR/P/173/15. 
geführt wurde. Neben den Beamten der Kolonialadministration schalteten sich auch Mediziner, Offiziere und Schiffskapitäne aktiv in die Debatte ein. Regen Anteil nahmen zudem Zeitungen, die sich an die Eliten der europäischen Community richteten. Während die Seeleute selbst nicht zu Wort kamen, wurden ihre Verhaltensweisen und mutmaßlichen Eigenschaften, ihr Gesundheitszustand, ihre sozioökonomische Lage sowie mögliche (Hilfs-)Maßnahmen in den Blick genommen. Die Diskussionen im Nachgang des Zyklons fanden Anschluss an breitere Debatten über die Rolle einer vermeintlich „niederen“ europäischen Klasse ${ }^{4}$ im kolonialen Gefüge. Über Fragen der Klassenzugehörigkeit hinaus wurden in diesem Zusammenhang auch kolonialrassistische Vorstellungswelten zivilisatorischer und moralischer Überlegenheit, Ideale einer Zivilisierungsmission und Motive eines bedrohlichen und korrumpierenden Indiens verhandelt.

Innerhalb der Debatte wurden die Seeleute in unterschiedliche Sicherheitszusammenhänge gestellt, gleichsam als potenzielle Bedrohung oder als in Gefahr betrachtet. Demgegenüber standen Kategorisierungen und Aushandlungsprozesse über Zugehörigkeiten und Abgrenzungen. Der vorliegende Beitrag betrachtet das Verhältnis verschiedener Konzeptionen und Produktionen von (Un-)Sicherheit und Differenz und fragt nach den Verknüpfungen und dem Zusammenwirken derartiger Prozesse sowie ihrem Verhältnis zu den Funktionsweisen kolonialer Herrschaft. Sicherheitsvorstellungen waren beispielsweise stark in koloniale Ideologien eingeschrieben und unterfütterten diese: Motive von Bedrohung und Gefahr wurden eingesetzt, um Herrschaftsansprüche zu begründen und zu legitimieren. Gleichzeitig ließ das Zusammenspiel aus Sicherheitsvorstellungen und Differenzkategorisierungen Widersprüche innerhalb der Herrschaftsideologien offen zu Tage treten. Gerade diese Widersprüche wurden wiederum als problematisch angesehen und rückten implizit ins Zentrum von Versicherheitlichungen. Eine reine Analyse der Diskussionen, wie sie in Schriften von Regierungsbeamten, medizinischen Publikationen und Presseberichten geführt wurden, reicht allerdings nicht aus, um die Reaktionen der Kolonialadministration im Anschluss an den Zyklon zu erklären. Zu diesem Zweck wird nach der Betrachtung zweier zentraler Argumentationslinien der Debatte ein Blick auf die Position der Seeleute im wirtschaftlichen System geworfen. Dies ist zum einen notwendig, um die Maßnahmen besser verstehen zu können. Zum anderen bringen die strukturellen Faktoren und materiellen Bedingungen eben jene widersprüchli-

4 Der Begriff lower classes wird hier als Quellenbegriff benutzt und mit „niedere“ oder „untere“ Klassen übersetzt. 
chen Konstellationen hervor, die in der Debatte verhandelt wurden. Wie der Zyklon vom Oktober 1864 zeigt, trugen diese Bedingungen ihrerseits Produktionen von (Un-)Sicherheit in sich. Abschließend wird deshalb die Möglichkeit genutzt, Auswirkungen kapitalistischer Funktionsweisen in einem kolonialen Umfeld zu diskutieren.

Der Beitrag schließt an Konzepte der kritischen Sicherheitsforschung an, die, über die Untersuchung von Sprechakten hinaus, auch solche strukturellen Faktoren in ihre Analyse einbeziehen, die entscheidend zur Ausgestaltung und Wirkmächtigkeit von Versicherheitlichungen beitragen (Balzacq 2011). Mit dem Fokus auf die Bevölkerungsgruppe der europäischen Seeleute knüptt der Beitrag zudem an Arbeiten von Sarmishta De (2008), Harald Fischer-Tiné (2009a, 2009b) und David Arnold (1979) an, die sich mit der Rolle ärmerer oder „subalterner“ Europäer*innen im kolonialen Indien im Allgemeinen befassen, aber auch die Lage der Seeleute in Kalkutta im Speziellen aufgreifen.

\section{Das Problem einer europäischen, lower class}

Selbst wenn es sich bei Britisch-Indien nie um eine ausgeprägte Siedlungskolonie handelte, verfügte es über eine signifikante und im Laufe des 19. Jahrhunderts stetig wachsende europäische Bevölkerung. Diese umfasste, neben den Beamten des Regierungsapparates und ihren Familienangehörigen, Kaufleute, Agenten europäischer Firmen, Pflanzer"innen und Ingenieure, aber auch Soldaten, Seeleute und Prostituierte ${ }^{5}$. Zeitgenössische Diskussionen über diese europäische Bevölkerung zeichneten sich durch ein deutlich erkennbares Klassenbewusstsein aus. Sowohl in Regierungsunterlagen als auch in veröffentlichten Schriften und Kommentaren ist ersichtlich, wie stark sich koloniale Eliten im Kollektiv von vermeintlich „niederen“ europäischen Klassen abgrenzten. Zu diesen lower classes wurden neben den bereits genannten Soldaten, Seeleuten und Prostituierten auch ungelernte Arbeiter*innen oder ehemalige Sträflinge aus Australien gezählt, die in Indien nach Arbeit suchten. Als Abgrenzungsmerkmale galten nicht nur die sozioökonomischen Positionen der unterschiedlichen Gruppen, sondern ebenso vermeintliche Charaktereigenschaften und habituelle Verhaltensweisen: Angehörige der „niederen“ Klassen wurden als lasterhaft, faul oder aufsässig beschrieben. Stets ihren eigenen Leidenschaften und Gelüsten

5 Die Bezeichnung wird hier als Quellenbegriff und damit als Fremdzuschreibung verwendet. 
folgend, waren sie angeblich leicht zu korrumpieren. Zudem pflegten sie aus Sicht der kolonialen Eliten einen schmutzigen und ungesunden Lebensstil (vgl. De 2008, S. 63ff.).

Zusätzlich zu diesen umfassenderen Klassenzuschreibungen waren Seeleute Teil gruppenspezifischer Narrative. Ein zentrales Motiv war hierbei die vermeintliche Nähe zu Alkohol und zur Trunkenheit. Das typische Bild, welches von einem (europäischen) Seemann nicht nur in Indien, sondern quasi global (vgl. Burton 2001; de Vries 2000) erstellt wurde, zeigte diesen als einen rohen Charakter, der sich, von den Entbehrungen und harschen Arbeitsbedingungen auf See gezeichnet, an Land diversen Vergnügungen und Lastern zuwandte. Zu diesen zählten der Besuch von Spelunken, zwielichtigen Tavernen und Bordellen, ein übermäßiger Alkoholkonsum und die Verwicklung in Tumulte und Schlägereien. Seeleute wurden in die Nähe von Kriminalität gerückt und bewegten sich am Rande städtischer Unterwelten. Ihr Verhalten galt zudem als laut und ungehobelt, rauflustig und schwer zu kontrollieren. Damit fügten sie sich nahtlos in die Charakterisierungen der lower classes ein. Selbst wohlwollende und mit den Seeleuten und ihrer Situation sympathisierende Beiträge zeichnen sich durch einen starken Paternalismus aus, welcher den Seeleuten eine lediglich geringe eigene Handlungsmacht und Entscheidungskompetenz zuschreibt. Sie galten als leichtfertige und unbedachte Klasse, die vor Verfehlungen bewahrt und auf die richtigen Wege geleitet werden musste. Diese Art der Bevormundung markierte und verfestigte die definierten Klassenunterschiede. ${ }^{6}$

Verbunden mit derartigen Bildern und Unterscheidungen waren Wahrnehmungen möglicher Probleme und Gefahren. Typisch hierfür ist eine Aussage des US-amerikanischen Generalkonsuls in Kalkutta aus dem Jahr 1865:

"Much sympathy has been expended upon the sailor, in many instances deservedly. For, as a class, undoubtedly they are defrauded and badly used, but in return they are insolent, wasteful, insubordinate, and slothful. [...] In self-defence, then, is it necessary to adopt measures effective and so possibly extreme, to prevent what otherwise will be

6 Siehe u.a. Chevers 1864; 'Christian Poverty and Vice in India - A Voluntary Poor Law Board', The Friend of India, 24. Mai 1866; 'The english Sailor in India', The Friend of India, 06. April 1865. 
a periodical and increasing nuisance, expense, and danger to this community."

Ähnlich äußerte sich der commissioner genannte leitende Beamte der Polizei von Kalkutta einige Monate nach dem Zyklon im Juni 1865. In einem Schreiben an die Regierung Bengalens bezeichnet er die aktuelle Lage der Seeleute als äußerst bedauerlich, warnt aber vor der Gefahr für Wohlergehen und Ruhe der Stadt, die hieraus erwachsen könne. Ohne Unterstützung würde man die Seeleute einer Welt von Hunger und Kriminalität aussetzen. ${ }^{8}$ Wie Harald Fischer-Tiné (2009a, S. 116ff.) herausgearbeitet hat, finden sich in den Polizeiberichten und Gerichtsakten Kalkuttas in den Jahren vor dem Zyklon in der Tat zahlreiche Hinweise auf europäische Seeleute. Zu den häufigsten Vergehen gehörten die Teilnahme an Schlägereien und gewalttätige Übergriffe, oftmals ausgelöst durch übermäßigen Alkoholkonsum oder Streitigkeiten über Geld in Gasthäusern und Bordellen. Darüber hinaus finden sich Berichte über Mord und Totschlag, zu deren Opfern in erster Linie die einheimische indische Bevölkerung zählte. Dementsprechend hoch war der Anteil der Seeleute unter europäischen Gefängnisinsassen in Kalkutta. Gemeinsam mit den Kosten möglicher Hilfsleistungen wurde gerade dieses Argument angeführt, um auch auf die finanzielle Bürde zu verweisen, die Seeleute für die britische Kolonialherrschaft bedeuteten. ${ }^{9}$ Seeleute galten demnach als Bedrohung für das körperliche Wohl der lokalen Bevölkerung, die Sicherheit von Leben und Eigentum, die städtische Ruhe und Ordnung und als Problem für die öffentlichen Kassen. Derartige Diagnosen blieben nicht auf Hafenstädte wie Kalkutta beschränkt. Unter der Überschrift „The New Colonist Danger“ warnte die in Kalkutta ansässige Zeitung The Friend of India im März 1866 beispielsweise vor den Gefahren, die von europäischen „Herumtreibern“10 im indischen Inland ausgingen, welche sich zu einem Teil aus arbeitslosen Seeleuten speisten: Vielerorts könne man keine englische Dame mehr alleine im Haus lassen oder den indischen Brauch fortführen, nachts Fenster und Türe offen zu halten. Immer wieder würden vor allem Inder*innen

7 Zitiert in Malleson, State of Sailors, S. 20. Für eine genaue Einordnung von Mallesons Bericht siehe Unterkapitel Seeleute und das gefährliche Andere.

8 V.H. Schalch an Secretary to the GovBengal, 30. Juni 1865, No 1094, BL IOR/P/173/15.

9 Siehe etwa John G. Reddie an Secretary to the GovBeng, 26. Juli 1865, No 1958, BL IOR/P/173/15.

10 Die originale englische Bezeichnung lautet 'loafer'. Siehe 'The New Colonist Danger', The Friend of India, 01. März 1866. 
Opfer von Übergriffen. Allzu oft müssten sie in Terror vor betrunkenen und lüsternen Soldaten oder Seeleuten fliehen.

Seeleute wurden jedoch auch auf eine andere Art als problematisch angesehen. In einem weiteren Artikel aus dem Jahr 1866 diagnostiziert The Friend of India etwa:

„From whatever point we view the lower classes of European Christians and their descendants in the large cities and stations in India, whether from the moral, political or industrial side, the conquering race, the white aristocracy, the civilizing power, have reason for shame."

Diese Sichtweise, welche die Seeleute als moralische und davon abgeleitet gesellschaftspolitische Gefahr für das Empire betrachtete, stellte keineswegs eine Ausnahme dar, sondern war ein zentraler Bestandteil des Blickes kolonialer Eliten auf europäische lower classes. Die aus Europa bekannten Diskurse (vgl. De 2008, S. 58ff.; Evans 2017, S. 165ff./339ff.; Mizutani 2009) über die „Respektabilität“ verschiedener Bevölkerungsschichten, über „deserving and undeserving poor" oder die "classes dangereuses", entfalteten in der Kolonie eine andere Wirkung, da sie auf rassistische Ideologien trafen, welche die koloniale Herrschaft unterfütterten. Für die kolonialen Eliten stellte die angebliche zivilisatorische Überlegenheit Europas ein wichtiges Fundament kolonialer Herrschaftsansprüche dar. Sie lieferte Begründungen für eine europäische Zivilisierungsmission, welche wiederum zur Legitimation imperialer Expansion und zum Selbstverständnis der Europäer"innen beitrug. Die vermeintlich „niederen“ Klassen passten kaum in dieses Bild. Ganz im Gegenteil standen sie mit ihren vermeintlichen moralischen Schwächen, ihrer Assoziation mit Trunkenheit, Unruhe, Kriminalität oder Krankheit eher der kolonisierten indischen Bevölkerung nahe. Dennoch waren sie eindeutig als europäisch und damit als Element des imperialen Projektes erkennbar. Die Anwesenheit einer derart „verwahrlosten“ Klasse an Europäer*innen untergrub einerseits rassistische Vorstellungen von Überlegenheit (De 2008, S.61; Fischer-Tiné 2009a, S. 81ff.), andererseits stellte sie die Kompetenz einer auf Ansehen und Prestige bedachten Kolonialadministration in Frage. Seeleute, die einen gewichtigen Teil der europäischen lower classes stellten, galten somit als Bedrohung für zentrale Annahmen, Grundsätze und Begründungen des kolonialen Unternehmens.

11 'Christian Poverty and Vice in India - A Voluntary Poor Law Board', The Friend of India, 24. Mai 1866. 
Das Beispiel zeigt in anschaulicher Art und Weise, wie die Produktion von Differenz zu einem Sicherheitsproblem werden konnte. Es wird deutlich, dass Abgrenzungen, die entlang verschiedener Differenzkategorien zum Zwecke der Herrschaftslegitimation vorgenommen wurden, in ihrer eigenen Logik potenzielle Sicherheitsrisiken aufwiesen. Dies galt vor allem dann, wenn unterschiedliche Abgrenzungskategorien zusammenwirkten beziehungsweise sich gegenüberstanden. Die auch aus Europa bekannten und von den britischen Eliten in Indien (re-)produzierten Klassenkonzeptionen trafen im kolonialen Raum auf die Funktionslogiken rassistisch unterfütterter imperialer Ideologien. Da die „niederen“ Klassen hier Teil der vermeintlich überlegenen europäischen Zivilisation waren, wurden ihre Eigenschaften und Verhaltensweisen als Problem betrachtet, gerade weil sie das zentrale Motiv der Überlegenheit in Frage stellten. In diesem Sinne bildeten sie eine Gefahr für die koloniale Ordnung.

\section{Seeleute und das gefährliche Andere}

„The principal causes of disease and death among sailors are bad feeding and bad air on shipboard, exposure to the effects of unhealthy climates, and, perhaps, above all, drunkenness and other evil habits when on shore. Of course these various causes of sickness often act together in ruining the health of those seaman who are long exposed to their united influence."

Mit diesen Worten beschrieb Norman Chevers (1864, S. 3), seinerseits Arzt und Leiter des Medical College in Kalkutta, in einem Vortrag im Januar 1864 den Gesundheitszustand europäischer Seeleute in der Stadt. Chevers' Ausführungen, welche später in Form eines Traktates veröffentlicht wurden, enthalten zahlreiche Elemente, die sich in den Debatten im Anschluss an den Zyklon wiederfinden. Darin wurden die Seeleute nicht nur als wichtiger Bestandteil des britischen imperialen Projektes und als christliche Glaubensgenossen identifiziert, sondern ebenso als eine besonders anfällige und gefährdete Personengruppe charakterisiert. Derartige Zuschreibungen ließen sie somit nicht nur als potenziell gefährliches Problem erscheinen, sondern ebenso als Gruppe, die Empathie und Hilfe benötigte und als schützenswert galt. Chevers steht hier beispielhaft für verschiedene Einzelpersonen, die versuchten, auf die Lage der Seeleute aufmerksam zu machen und mögliche Initiativen zu deren Verbesserung einzubringen. Die britische Kolonialregierung nahm derartige Vorstöße und Initiativen zwar durchaus auf, ein ausgeprägtes Engagement ist dennoch kaum er- 
kennbar. Im April 1864 legte beispielsweise Vizekönig John Lawrence im Rahmen einer öffentlichen Zeremonie den Grundstein für ein neues Sailors' Home in Kalkutta. ${ }^{12}$ Die Einrichtung sollte als eine sichere und ordentliche Unterkunft für Seeleute an Land dienen, sodass diese nicht obdachlos zurückblieben oder auf als unsauber und höchst problematisch wahrgenommene Herbergen - so genannte lodging-houses - zurückgreifen mussten. Das neue Sailors' Home, welches ohnehin maßgeblich durch Spendengelder finanziert worden war, wurde jedoch von Baubeginn an für seine mangelnde Größe kritisiert: Maximal die Hälfte aller normalerweise in Kalkutta verbleibenden arbeitslosen europäischen Seeleute konnte hier Unterkunft finden. Auch in Zeitungen wurde noch 1865 und 1866 kritisiert, dass den Seeleuten in ihrer Lage durch die Regierung und in der Öffentlichkeit zu wenig Beachtung geschenkt werde. ${ }^{13}$

Selbst im Nachgang des Zyklons blieb die Administration in einer passiven und reaktiven Rolle verhaftet. Eine breitere Untersuchung zur Lage der Seeleute wurde beispielsweise nicht unmittelbar durch die Kolonialregierung initiiert, sondern, in Person von Major G.B. Malleson, durch ein Mitglied der Sanitary Commission, einem kurz zuvor geschaffenen Organ mit lediglich beratender Funktion und Kompetenz, an diese herangetragen. ${ }^{14}$ Malleson knüptte in seinem Bericht ${ }^{15}$ wiederum ausdrücklich an Chevers' Überlegungen an. Beide beobachteten eine außergewöhnlich hohe Sterblichkeitsrate unter europäischen Seeleuten in Kalkutta und führten diese auf die Bedingungen zurück, denen die Seeleute während ihres Aufenthaltes in der Stadt ausgesetzt waren. ${ }^{16}$ Gemeinsam erlangten ihre Darstellungen eine große öffentliche Aufmerksamkeit und prägten die Debatte im Anschluss an den Zyklon. Die Schilderungen und Einschätzungen zur problematischen Lage der Seeleute wurden durchaus schockiert zur Kenntnis genommen und immer wieder benutzt, um Kritik zu üben, Sympathien zu wecken oder um Spenden zu werben. ${ }^{17}$

12 Siehe 'The Week', The Friend of India, 14. April 1864.

13 Siehe z.B. 'The english Sailor in India', The Friend of India, 06. April 1865; 'Our Sailors', The Friend of India, 30. August 1866.

14 J.P. Walker, Sec SanCommission, an Secy to the GovBengal, 28. Juli 1865, No317, BL IOR/P/173/15.

15 G.B. Malleson: The State of the Sailors in Calcutta, 1865. Enthalten in BL, IOR/P/437/29, India Proceedings, Marine, 1866.

16 Chevers Angaben zufolge lag die Sterblichkeitsrate unter Seeleuten in Kalkutta knapp zehnmal höher als in britischen Häfen. Vgl. Chevers 1864, S. 40.

17 Siehe u.a. 'The English Sailor in India, The Friend of India, 06.04.1865; 'Our Sailors', The Friend of India, 11.01.1866; 'Christian Poverty and vice in India - 
Was machte die Situation der Seeleute so problematisch und gefährlich? Bereits das obige Zitat von Chevers verweist auf unterschiedliche Sicherheits- und Gesundheitsprobleme. Die habituelle Trunkenheit, bereits im vorherigen Abschnitt als problematisches Kennzeichen der Seeleute diskutiert, galt auch als ein großes Gesundheitsrisiko. Verschärft wurde dies in den Augen der Beobachter*innen dadurch, dass der in Kalkutta verkaufte Alkohol als "giftig“ angesehen ${ }^{18}$ und der Hang der Seeleute zum Trinken von dritten Personen ausgenutzt wurde. In Mallesons Bericht heißt es hierzu beispielsweise:

„[...] that the ignorant, inexperienced, unlettered seaman is suffered to roam all over the place with no protection from the sun, probably little knowing its power in this country, that temptations to drink are constantly before him, aggravated by the climate; that he is cared for by the Boarding Masters only so long as they are able to make a profit out of him; that for this purpose they encourage him to drink and get drunk on their premises; and that when his funds are exhausted they turn him out, beggared, into the streets." (Malleson 1865, S. 11)

Deutlich wird außerdem, wie die klimatischen Bedingungen Bengalens als massives Gesundheitsproblem beschrieben werden. Eine unbarmherzige Sonne, große Hitze, aber auch die Regenfälle der Monsunzeit wurden immer wieder angeführt. Gleiches gilt für verschiedene Krankheiten: Allen voran Cholera und durch Infektionen verursachte Durchfallerkrankungen wurden als tödliche Bedrohung eingestuft. Neben Alkohol, Krankheiten und den klimatischen Bedingungen galt die Prostitution als Problem und Gefahrenquelle. So schrieb der britische Kapitän Henry Toynbee beispielsweise in einem Brief an den Secretary of State for India:

„When they go on shore for recreation, their only resting place is Flag Street, inhabited by brothel-keepers, prostitutes, and crimps of the lowest order, full of the surest and foulest temptations for the seaman after a long voyage. Let a seaman with the best intentions land here merely for recreation, and he invariably yields to temptation, loses all self-respect; and, if a youngster, he begins that downward career which ends in the ruin of so many of our seamen; and, if a grown man, he contracts a loathsome disease and becomes a burden on the ship, crew,

A Voluntary Poor Law Board', The Friend of India, 24.05.1866; 'Our Sailors', The Friend of India, 30.08.1866.

18 Siehe Chevers 1864, S. 36ff; Malleson 1865, S. 1 ff. 
and the country, and has finally to be sent home at the Government expense." 19

Analog zu den oben diskutierten Klassenzuschreibungen wurden auch Fragen der Gesundheit häufig mit Themen wie Moral und Ordnung verknüpft. Krankheit und Schmutz galten als Ausdruck charakterlicher Verwahrlosung. Prostituierte wurden so nicht nur durch ihre Assoziation mit Krankheit, insbesondere der Syphilis, zum Teil einer bedrohlichen Kulisse, sondern ebenso durch ihre vermeintliche Amoralität, welche angeblich zum Verfall der Seeleute führe. Letztere wurden also als stark bedrohtes Referenzobjekt von Sicherheitsdenken wahrgenommen. Die entsprechenden Gefahren kamen von außen, aus der indischen Umgebung, den natürlichen Bedingungen Bengalens und durch den Umgang mit vermeintlich schädlichen Personengruppen.

Dieses Motiv der Gefährlichkeit Indiens verdeutlicht, wie stark Beiträge im Rahmen der Debatte an zentrale imperiale Ideologien anknüpften. In erster Linie zählt hierzu eine sich im Laufe des 19. Jahrhunderts zunehmend verfestigende, rassistische Dichotomisierung von Europa und Indien. Wie unter anderem Mark Harrison (1999) argumentiert, finden sich in der europäischen Literatur des 17. und 18. Jahrhunderts, welche sich mit Indien auseinandersetzt, zunächst kaum strikte und fixierte Trennungen zwischen einem europäischen „Eigenen“ und indischen „Anderen“. Dies änderte sich nach 1800 zusehends. Mit dem Aufkommen eines biologisch fundierten Verständnisses von Rasse und einer territorialen und administrativen Konsolidierung der kolonialen Herrschaft nahmen die britischen Eliten eine schärfere Abgrenzung zur indischen Bevölkerung und Umwelt vor (vgl. Metcalf 1994, S. 44 und 66ff.; Nightingale 2012, S. $111 \mathrm{ff}$.). Während es vor 1800 durchaus einen verhaltenen Optimismus hinsichtlich einer möglichen europäischen Akklimatisierung gegeben hatte (vgl. Harrison 1999, S. 3), schlug dieser zunehmend in Pessimismus um. Wie die obige Debatte zeigt, wurde Indien mit Schrecken betrachtet und galt als Hort der Krankheit, des Schmutzes und des Verfalls. Indem die europäisch und christlich definierten Seeleute als schützenswerte Personen vor einer bedrohlichen Umwelt und Umgebung dargestellt wurden, konnten koloniale Ideologien weiter transportiert und verfestigt werden.

Das Prisma „Natur“ eignete sich auf eine weitere Art und Weise, um eine Abgrenzung zwischen Europa und Indien zu forcieren. Der Fokus auf die große Bedeutung von Krankheiten, Hitze oder Monsunregen ließ

19 Captain H. Toynbee an Secy of State for India, 27. März 1868, No25, BL IOR/P/434/45. 
Indien zu einem den Elementen ausgelieferten Land werden; einem Land, welches sich nicht durch eine überlegene Kultur auszeichnete, sondern sich den Kräften der Natur unterordnen musste. Demgegenüber stand ein modernes Europa, überzeugt von einem Bewusstsein, eben jene Natur besser verstanden und überwunden zu haben, und dementsprechend auf einer höheren zivilisatorischen Stufe zu stehen (vgl. Arnold 2005). Ein derartiges Ungleichgewicht, die diagnostizierte „Rückständigkeit“ Indiens und seine vermeintlich inhärenten Schwächen ließen eine Kolonisierung geradezu zwangsläufig erscheinen. Verortet wurde diese angenommene zivilisatorische Höherwertigkeit zudem auf einer moralisch-ethischen Ebene. Der Indien attestierten moralischen Verwahrlosung wurde der überlegene britische character entgegengesetzt (vgl. Cain 2018). Die Seeleute drohten durch ihre Eigenschaften und Verhaltensweisen dieses Bild der Europäer zu untergraben. Der Einfluss der indischen Umwelt bot nun jedoch eine mögliche Erklärung: Sie verführte und korrumpierte die Seeleute, ließ sie vom richtigen Pfad abkommen und setzte sie Ruin und Verfall aus. Selbst für die Gewalt der Seeleute fand sich eine Erklärung. So führt The Friend of India zum Verhalten Europäer „niederer“ Klassen an:

„If subject to the restraints of law as in England he would not yield to those temptations offered by effeminate natives and a hot climate, which so often end in crime and not unfrequently in murder." 20

Besonders die Bevölkerung Bengalens wurde von britischer Seite immer wieder als „verweiblicht“ und daraus abgeleitet „schwächlich“ abgetan (Sinha 1995). Ohne an dieser Stelle näher auf die Gender-Dynamiken und Implikationen derartiger Zuschreibungen einzugehen, soll kurz herausgestellt werden, dass solche Einordnungen nicht nur versuchten, eine Überlegenheit der Europäer*innen aufrecht zu erhalten, sondern ebenso das Motiv unterstrichen, die genuine Schwäche Indiens beziehungsweise der indischen Bevölkerung sei für ihre Kolonisierung verantwortlich.

Deutlich wird mit Betrachtung der Debatte somit insgesamt, wie stark Sicherheitslogiken in imperiale Ideologien eingebunden waren. Die Konstruktion Indiens als das gefährliche Andere stützte sich einerseits auf bestehende Differenzwahrnehmungen. Andererseits stellte gerade das Element der Bedrohung einen zentralen Bestandteil der vermeintlichen Andersartigkeit dar. In diesem Sinne konnten kolonialrassistische Denkweisen verfestigt sowie Begründungen und Rechtfertigungsstrategien für die andauernde Kolonisierung Indiens weitergetragen werden. Die Versi- 
cherheitlichungen wurden somit nicht nur genutzt, um bestimmte Differenzzuschreibungen zu essentialisieren, sondern ebenso um sich der Herrschaftsansprüche und -vorstellungen zu vergewissern. Für die Seeleute, das vordergründige Referenzobjekt der Debatte, bot ihre Identifizierung als Teil der Kolonisierenden wiederum die Möglichkeit, dass ihnen Sympathie und in gewissem Maß Hilfe zu Teil wurde. Indischen Seeleuten, welche ebenso von den Auswirkungen des Zyklons betroffen waren, wurde eine derartige Aufmerksamkeit nicht zuteil.

\section{Fazit: (Un-)Sicherheit im kapitalistischen System}

In den Diskussionen um die Lage der Seeleute wurden die Fluktuation auf dem Arbeitsmarkt sowie das Verhalten der Schiffskapitäne eher im Hintergrund thematisiert. Beides trug jedoch elementar zur Situation der Seeleute bei und verweist zugleich auf deren Position im ökonomischen System. Gemeinsam mit verwandten Berufsgruppen wie Hafen- oder Werftarbeiter*innen galt der Berufsstand der Seeleute als besonders anfällig für wirtschaftliche Schwankungen, vor allem aufgrund der Kurzfristigkeit ihrer Arbeitsverhältnisse. Schiffsmannschaften wurden in der Regel für eine Überfahrt oder eine Hin- und Rückreise beschäftigt, Hafenarbeiter"innen oftmals gar nur Tag für Tag (vgl. Kenefick 2000). Dementsprechend konnten nicht nur langfristige ökonomische Krisen, sondern auch wetterbedingte, saisonale Unterschiede enorme Auswirkungen entfalten. Für die Seeleute bedeutete dies ein unstetes Leben von Lohn zu Lohn und ohne Absicherung. In nahezu jeder Hafenstadt fand sich daher eine variierende Zahl arbeitsloser Seeleute. Die kolonialen Behörden Kalkuttas waren sich dieses Umstandes durchaus bewusst, fassten Seeleute ohne Beschäftigung zu einem gewissen Grad jedoch als normalen Teil der floating population ihrer Stadt auf. ${ }^{21}$

Aktiv begünstigt wurde diese Situation durch die Entlassungspolitik der Schiffskapitäne. Im Hafen von Kalkutta angekommen, entließen diese oftmals ihre Mannschaften, um keine Gehälter zahlen zu müssen, solange das Schiff vor Anker lag, und für die Weiter- oder Rückfahrt eine neue Mannschaft mit geringeren Bezügen beschäftigen zu können. Wenngleich diese Praxis im Prinzip unter Beaufsichtigung der Regierung stand und durchaus kritisch beäugt wurde, können Versuche der Regierung hier stärker einzuwirken allenfalls als halbherzig bezeichnet werden. Gesetzli-

21 A. Eden an Master Attendant, 16.12.1864, No 5817A, BL IOR/P/173/15. 
che Regelungen wurden nicht vollständig ausgeschöpft oder waren leicht zu umgehen. ${ }^{22}$ Immer wieder finden sich, unter anderem in Mallesons Bericht, Schilderungen über Kapitäne, die außergewöhnlich harte Arbeitsanweisungen und Disziplinierungsmaßnahmen dazu nutzten, um Seeleute zur "gegenseitigen“ Vertragsauflösung zu bringen oder Reaktionen wie Streiks oder Arbeitsverweigerungen zu provozieren, welche dann wiederum mit Entlassung oder eventuell sogar Freiheitsstrafen geahndet werden konnten. ${ }^{23}$ Derartige Episoden unterstreichen die konstant prekäre sozioökonomische Position der Seeleute. Krisen wie der Zyklon, welcher die Beschäftigungsmöglichkeiten reduzierte und die Dauer von Arbeitslosigkeit und Aufenthalt in Kalkutta verlängerte, verschärften die Situation.

Vor diesem Hintergrund sind die Maßnahmen zu sehen, die im unmittelbaren Nachgang des Zyklons diskutiert und durchgeführt wurden. An vorderster Stelle stand das schlichte Entsenden der Seeleute nach Großbritannien. Nach den Bestimmungen des Merchant Seaman Act von 1856 konnten in Not geratene Seeleute auf Kosten der Regierung zurück in das Vereinigte Königreich geschickt werden. Wenngleich diese Maßnahme vor allem als eine erste Reaktion zur Anwendung kam, wurde sie von der Regierung Bengalens schnell mit äußerster Vorsicht betrachtet. Kritisch gesehen wurde etwa der hierfür nötige finanzielle Aufwand, über den sich neben einigen Kolonialbeamten auch eine Versammlung der Schiffskapitäne beschwerte. ${ }^{24}$ Dabei verband sich die Frage der Kosten häufig mit der Sorge, die falschen Seeleute nach Großbritannien zu schicken. So wies die Regierung die zuständigen Beamten explizit an, genau darauf zu achten, nur jene Personen nach Hause zu senden, die nachweislich durch den Zyklon arbeitslos geworden waren. Alle anderen, insbesondere eine Gruppe vermeintlich hartgesottener Bettler, kämen für Hilfe durch die Regierung nicht in Frage. ${ }^{25}$ Mit Blick auf Letztere wurde stattdessen ein vagrancy law nach britischem Vorbild in die Diskussion eingebracht. Die Beamten schlossen demnach nicht nur an die ideologischen Differenzierungen armer Europäer*innen in aufrichtige und berechtigte Empfänger*innen von Hilfe auf der einen und unwürdige, unverdiente Personen auf der anderen Seite an, sondern ebenso an Denkweisen, welche bestimmte Arten der Arbeits- und Obdachlosigkeit kriminalisierten. Eine Unterstützung ärmerer Bevölkerungsschichten ohne weitere Unterscheidungen wurde zur Mitte

22 Cecil Beadon an John Lawrence, 07. August 1865, No68, BL Mss Eur F90/48.

23 Siehe Malleson 1865, S. 5ff.

24 Siehe H. Toynbee an R.N. Cust, 13.12.1864, BL IOR/P/173/15.

25 A. Eden an Master Attendant, 16.12.1864, No 5817A, BL IOR/P/173/15. 
des 19. Jahrhunderts im Vereinigten Königreich nicht zuletzt aus dem Grund als problematisch betrachtet, als diese zur Faulheit verleiten und die Verelendung der Bevölkerung weiter begünstigen würde (vgl. De 2008, S. 61; Evans 2017, S. 119ff.). Vielmehr galt es, im Sinne kapitalistischer Interessen eine möglichst breite Basis potenzieller Arbeiter*innen aufrechtzuerhalten beziehungsweise bereitzustellen. Diese Denkweise zeigte sich auch im vorliegenden Beispiel. Die gemeinsame Überzeugung teilend, dass eine gewisse "Reserve“ an arbeitslosen Seeleuten notwendig sei, um das Funktionieren des Hafens von Kalkutta zu gewährleisten, wurde in Regierungskreisen über das nötige Mindestmaß diskutiert. Die zuständigen Stellen wiederum erhielten den Auftrag, eine entsprechende Zahl arbeitsloser Seeleute in Kalkutta zu erhalten. ${ }^{26}$ Obwohl zeitgenössische Studien beschrieben, dass dies zu einer hohen Sterblichkeitsrate führte, war die Kolonialregierung demnach bereit, die prekäre Position der Seeleute in Kauf zu nehmen und sie zugunsten anderer Interessen den Konsequenzen auszusetzen.

Das Beispiel der Seeleute zeigt in kleinem Rahmen die Bedeutung der Kategorien (Un-)Sicherheit und Differenz für die Funktionsweise des ökonomischen Systems: Beide fungierten als aufeinander bezogene Ko-Konstituenten der Klassenposition der Seeleute. Die fragile Lage der Seeleute mit ihrer konstanten Abhängigkeit von äußeren Bedingungen und der Ungewissheit über Beschäftigungsmöglichkeiten war ein Kennzeichen möglicher Unterschiede und damit ein wichtiges Merkmal der Abgrenzung. Koloniale Eliten waren den lokalspezifischen Witterungsbedingungen und verschiedenen Krankheiten deutlich weniger ausgesetzt beziehungsweise konnten ihnen einfacher entgehen. Für die Seeleute stellte ihre Situation hingegen gleichsam ein Definitionsmerkmal und eine Folge ihrer sozioökonomischen Position dar. In diesem Sinne handelte es sich bei der problematischen Anwesenheit einer europäischen lower class keineswegs um einen systemischen Fehler. Vielmehr war sie Konsequenz der kapitalistischen Wirtschaftsweise. Der Zyklon vom Oktober 1864 ließ die damit einhergehenden Unsicherheiten und Risikofaktoren offen zu Tage treten und verlieh den strukturellen Problemen eine größere Sichtbarkeit und höhere Dringlichkeit.

Die Versicherheitlichungen und Abgrenzungen, die in der Debatte über die Lage der Seeleute entlang rassistischer und klassistischer Kriterien vorgenommen wurden, weisen auf strukturelle Widersprüche hin, die der

26 A. Eden an Master Attendant, 16.12.1864, No 5817A, BL IOR/P/173/15; John G. Reddie an Secy GovBengal, 29.12.1864, No 4188, BL IOR/P/173/15. 
kolonialen Herrschaft innewohnten. Diese Widersprüchlichkeit spiegelte sich nicht nur in der ambivalenten Position der europäischen Seeleute zwischen Referenzobjekt und potenzieller Bedrohung wider, sondern rückte selbst ins Zentrum der Versicherheitlichungen. Differenzkategorisierungen, wie sie etwa mit Blick auf eine vermeintliche zivilisatorische Überlegenheit Europas vorgenommen wurde, fungierten als Basis und Rechtfertigung kolonialer Herrschaft. Derartige Vorstellungen drohten durch die Seeleute oder, genauer, durch die ihnen zugeschriebenen Eigenschaften und Verhaltensweisen sowie ihre prekäre Situation untergraben zu werden. Hierdurch hervorgerufene Unsicherheiten zeigten sich dementsprechend auch darin, dass die kolonialen Eliten jene Denkweisen und Differenzzuschreibungen, die den kolonialen Ideologien zugrunde lagen, bewusst betonten, um sich ihrer eigenen Position zu vergewissern. Die Lage der Seeleute wiederum resultierte aus ihrer Position im ökonomischen System. Während an dieser Stelle also unterschiedliche Konzeptionen und Produktionen von (Un-)Sicherheit und Differenz zusammenwirkten, deutet sich ein grundlegendes Sicherheitsdilemma der kolonialen Herrschaft an. Die Widersprüche entstanden aus materiellen Bedingungen und ideologischen Vorstellungen, die in die Herrschaft selbst eingeschrieben waren. In diesem Sinne waren sie strukturell angelegt und ohne Weiteres kaum aufzulösen. Kleinere Ansätze zeigten sich im vorliegenden Beispiel, wenn über mögliche Verbesserungen der Lage der Seeleute gesprochen wurde. Diese blieben jedoch oberflächlich verhaftet und wurden von anderen Interessen überlagert. Mit Blick auf Fragen der Sicherheitsforschung wird deutlich, dass Debatten und Argumente im Rahmen von Versicherheitlichungen in ihren strukturellen Umgebungen gesehen und durch Gesellschaftsanalysen unterfüttert werden müssen. Ohne dies wäre etwa die hier betrachtete Situation der Seeleute nicht ausreichend zu erklären.

\section{Literatur}

Arnold, David (1979): European Orphans and Vagrants in India in the Nineteenth Century. In: The Journal of Imperial and Commonwealth History 7, H. 2, S. 104-127.

Ders. (2005): The Tropics and the Traveling Gaze. India, Landscape and Science 1800-1856. New Delhi: Combined Academic.

Balzacq, Thierry (2011): A Theory of Securitization. Origins, core Assumptions, and Variants. In: Balzacq, Thierry (Hrsg.): Securitization Theory. How security Problems emerge and dissolve. Abingdon u. New York: Routledge, S. 1-30. 
Burton, Valerie (2001): Boundaries and Identities in the Nineteenth Century English Port: Sailortown Narratives and Urban Space. In: Gunn, Simon/ Morris, Robert John (Hrsg.): Identities in Space. Contested Terrains in the Western City since 1850. Aldershot: Ashgate, S. 137-151.

Cain, Peter (2018): Character, Ethics and Economics. British Debates on Empire, 1860-1914. London: Routledge.

Chevers, Norman (1864): On the Preservation of the Health of Seamen, especially of those frequenting Calcutta and the other Indian Ports. Calcutta.

De, Sarmishta (2008): Marginal Europeans in colonial India, 1860-1920. Kolkata: Thema.

de Vries, David (2000): The Construction of the Image of Dock Labour. In: Davies, Sam/Davis, Colin J./de Vries, David/ van Voss, Lex Heema/Hesselink, Lidewij/ Weinhauer, Klaus (Hrsg.): Dock Workers. International Explorations in Comparative Labour History, 1790-1970, Aldershot: Ashgate 2000, S. 681-708.

Evans, Richard J. (2017): The Pursuit of Power. Europe 1815-1914. London: Penguin.

Fisher-Tiné, Harald (2009a): Low and Licentious Europeans. Race, Class and 'White Subalternity' in Colonial India. New Delhi: Orient Blackswan.

Ders. (2009b): Flotsam and Jetsam of the Empire? European Seamen and Spaces of Disease and Disorder in Mid-nineteenth Century Calcutta. In: Tambe, Ashwini/ Fisher-Tiné, Harald (Hrsg.): The Limits of British Colonial Control in South Asia. Spaces of Disorder in the Indian Ocean Region. Abingdon u. New York: Routledge, S. 121-152.

Gastrell, J.E./Blanford, H.F. (1866): Report on the Calcutta Cyclone on the $5^{\text {th }}$ of October 1864. Calcutta.

Harrison, Mark (1999): Climates \& Constitutions. Health, Race, Environment and British Imperialism in India, 1600-1850. Oxford: Oxford University Press.

Kenefick, William (2000): Rebellious and Contrary. The Glasgow Dockers, 18531932, Edinburgh: Tuckwell Press.

Metcalf, Thomas R. (1994): Ideologies of the Raj. Cambridge: Cambridge University Press.

Mizutani, Satoshi (2009): 'Degenerate whites' and their Spaces of Disorder. Disciplining racial and class Ambiguities in colonial Calcutta (c.1880 - 1930). In: Tambe, Ashwini/Fisher-Tiné, Harald (Hrsg.): The Limits of British Colonial Control in South Asia. Spaces of Disorder in the Indian Ocean Region. Abingdon u. New York: Routledge, S. 153-192.

Nightingale, Carl H. (2012): Segregation. A Global History of Divided Cities. Chicago: The University of Chicago Press.

Sinha, Mrinalini (1995): Colonial Masculinity. The „manly Englishman“ and the „effeminate Bengali“ in the late Nineteenth Century. Manchester: Manchester University Press. 
